\title{
Platelet desialylation is a novel mechanism and a therapeutic target in thrombocytopenia during sepsis: an open- label, multicenter, randomized controlled trial
}

Mei-feng $\mathrm{Li}^{1,2+}$, Xiao-li $\mathrm{Li}^{2+}$, Kai-liang Fan ${ }^{1,3+}$, Ying-yi $\mathrm{Yu}^{1+}$, Jing Gong ${ }^{4}$, Shu-ying Geng ${ }^{5}$, Ya-feng Liang ${ }^{2}$, Ling Huang ${ }^{6}$, Ji-hua Qiu' ${ }^{1}$, Xing-han Tian², Wen-ting Wang ${ }^{2}$, Xiao-lu Zhang ${ }^{2}$, Qing-xia Yu ${ }^{2}$, Yuan-feng Zhang ${ }^{2}$, Peng Lin², Li-na Wang ${ }^{5}$, Xin Li ${ }^{1}$, Ming Hou ${ }^{1,7}$, Lu-yi Liu ${ }^{2^{*}}$ and Jun Peng ${ }^{1 *}$

\begin{abstract}
Background: Studies in murine models suggested that platelet desialylation was an important mechanism of thrombocytopenia during sepsis.

Methods: First, we performed a prospective, multicenter, observational study that enrolled septic patients with or without thrombocytopenia to determine the association between platelet desialylation and thrombocytopenia in patients with sepsis, severe sepsis, and septic shock. Gender- and age-matched healthy adults were selected as normal controls in analysis of the platelet desialylation levels (study I). Next, we conducted an open-label randomized controlled trial $(R C T)$ in which the patients who had severe sepsis with thrombocytopenia (platelet counts $\leq 50 \times 10^{9} / L$ ) were randomly assigned to receive antimicrobial therapy alone (control group) or antimicrobial therapy plus oseltamivir (oseltamivir group) in a 1:1 ratio (study II). The primary outcomes were platelet desialylation level at study entry, overall platelet response rate within 14 days post-randomization, and all-cause mortality within 28 days post-randomization. Secondary outcomes included platelet recovery time, the occurrence of bleeding events, and the amount of platelets transfused within 14 days post-randomization.
\end{abstract}

Results: The platelet desialylation levels increased significantly in the 127 septic patients with thrombocytopenia compared to the 134 patients without thrombocytopenia. A platelet response was achieved in 45 of the 54 patients in the oseltamivir group (83.3\%) compared with 34 of the 52 patients in the control group (65.4\%; $P=0.045$ ). The median platelet recovery time was 5 days (interquartile range 4-6) in the oseltamivir group compared with 7 days (interquartile range 5-10) in the control group $(P=0.003)$. The amount of platelets transfused decreased significantly in the oseltamivir group compared to the control group $(P=0.044)$. There was no difference in the overall 28-day mortality regardless of whether oseltamivir was used. The Sequential Organ Failure Assessment score and platelet recovery time were independent indicators of oseltamivir therapy. The main reason for all of the mortalities was multiple-organ failure.

(Continued on next page)

\footnotetext{
*Correspondence: luyi65166@126.com; junpeng88@sina.com.cn

${ }^{\dagger}$ Equal contributors

${ }^{2}$ Intensive Care Unit, and Clinical Laboratory, Yantai Yuhuangding Hospital

Affiliated to Qingdao University, Yantai 264000, Shandong, China

'Department of Hematology, Qilu Hospital, Shandong University, Jinan

250012, China

Full list of author information is available at the end of the article
} 
(Continued from previous page)

Conclusions: Thrombocytopenia was associated with increased platelet desialylation in septic patients. The addition of oseltamivir could significantly increase the platelet response rate, shorten platelet recovery time, and reduce platelet transfusion.

Trial registration: Chinese Clinical Trial Registry, ChiCTR-IPR-16008542.

Keywords: Sepsis, Thrombocytopenia, Oseltamivir, Desialylation, Platelet

\section{Background}

Sepsis is a systemic, deleterious host response to infection leading to severe sepsis and possibly septic shock as defined by the Surviving Sepsis Campaign guidelines $[1,2]$. Thrombocytopenia is a common finding in sepsis, which is associated with adverse outcomes in patients admitted to the intensive care unit (ICU) [3-5]. In patients with sepsis, thrombocytopenia may not merely be a marker of disease severity but even play a causative role in increased sepsis morbidity and mortality $[6,7]$. Thus, the correction of thrombocytopenia becomes a key issue for clinicians during the treatment of sepsis.

The mechanisms of septic thrombocytopenia are not well established and might include bone marrow suppression, immune destruction, disseminated intravascular coagulation (DIC), and platelet chemotaxis induced by inflammatory mediators [8-18]. Grewal et al. found that the marked thrombocytopenia in Streptococcus pneumonia sepsis was not mediated by the pathogen itself or the consumption in the process of DIC but due to the Ashwell-Morell receptor (AMR)-dependent clearance of platelets desialylated by NanA neuraminidase $[19,20]$. Currently, with the exception of acute platelet transfusion when necessary, there is no effective method to prevent thrombocytopenia.

Sialic acid is a natural sugar acid compound that exists widely across organisms [21]. The ends of glycoprotein chains on platelet membranes are all covered by sialic acid to protect the platelets from being destroyed. Sialidases, also known as neuraminidases, are sialic acid-releasing exoglycosidases that catalyze the removal of terminal sialic acids from sialosides and sialoglycoconjugates in nature [22]. Sialidase widely exists in viruses, bacteria, and mammalian cells [23]. Additionally, resting platelets contain an internal pool of sialidases which are released upon activation from any cause or after a pathogenic bacteriumreleased new sialidase is introduced into the serum. The endogenous and exogenous sialidases hydrolyze terminal sialic acid moieties from platelet glycoproteins [19, 24]. Several studies in murine models suggested that sialidases released or upregulated during infections hydrolyze sialic acid from platelet glycoproteins [25, 26]. Desialylation leads to the exposure of $\beta$-galactose residues on platelets, which can be recognized by AMRs on hepatocytes and eventually results in platelet phagocytosis in the liver
[27, 28]. It becomes increasingly evident that desialylation is responsible for, at least in part, the pathogenesis of thrombocytopenia in many diseases and the clearance of transfused platelets after storage [29]. Desialylation-induced platelet removal could possibly be circumvented by adding sialidase inhibitors during sepsis or after refrigeration $[19,30,31]$.

Oseltamivir, also known as Tamiflu, is an extensively used and clinically effective anti-influenza drug. It is a viral sialidase inhibitor that prevents the release of progeny virions, thus limiting the spread of infection [32]. Several studies indicate the feasibility that oseltamivir can be used for the treatment of infection-associated thrombocytopenia. Oseltamivir could elevate platelet counts in the treatment of pediatric as well as adult flu patients with thrombocytopenia $[30,33]$. Recently, we reported the successful treatment with oseltamivir phosphate of a patient with chronic immune thrombocytopenia (ITP) and influenza [34]. Hence, we hypothesized that thrombocytopenia was associated with increased platelet desialylation in septic patients and that the addition of oseltamivir to an antibiotic therapy would improve the clinical outcome in severe sepsis patients with thrombocytopenia. The study demonstrates that platelet desialylation is a novel mechanism in thrombocytopenia during sepsis. It would provide valuable theoretical foundation and therapeutic targets for the clinical treatment of septic thrombocytopenia.

\section{Methods \\ Study design}

First, we performed a prospective, multicenter, observational study that enrolled septic patients with or without thrombocytopenia to determine the association between platelet desialylation and thrombocytopenia in patients with sepsis, severe sepsis, and septic shock. Gender- and age-matched healthy adults were selected as normal controls in analysis of the platelet desialylation levels (study I). Next, we conducted an open-label randomized controlled trial (RCT) in which the patients from study I who had severe sepsis with thrombocytopenia (platelet counts $\leq 50 \times 10^{9} / \mathrm{L}$ ) were randomly assigned to receive antimicrobial therapy alone (control group) or antimicrobial therapy plus oseltamivir (oseltamivir group) in a 1:1 ratio (study II). The study flowchart is shown in Fig. 1. 


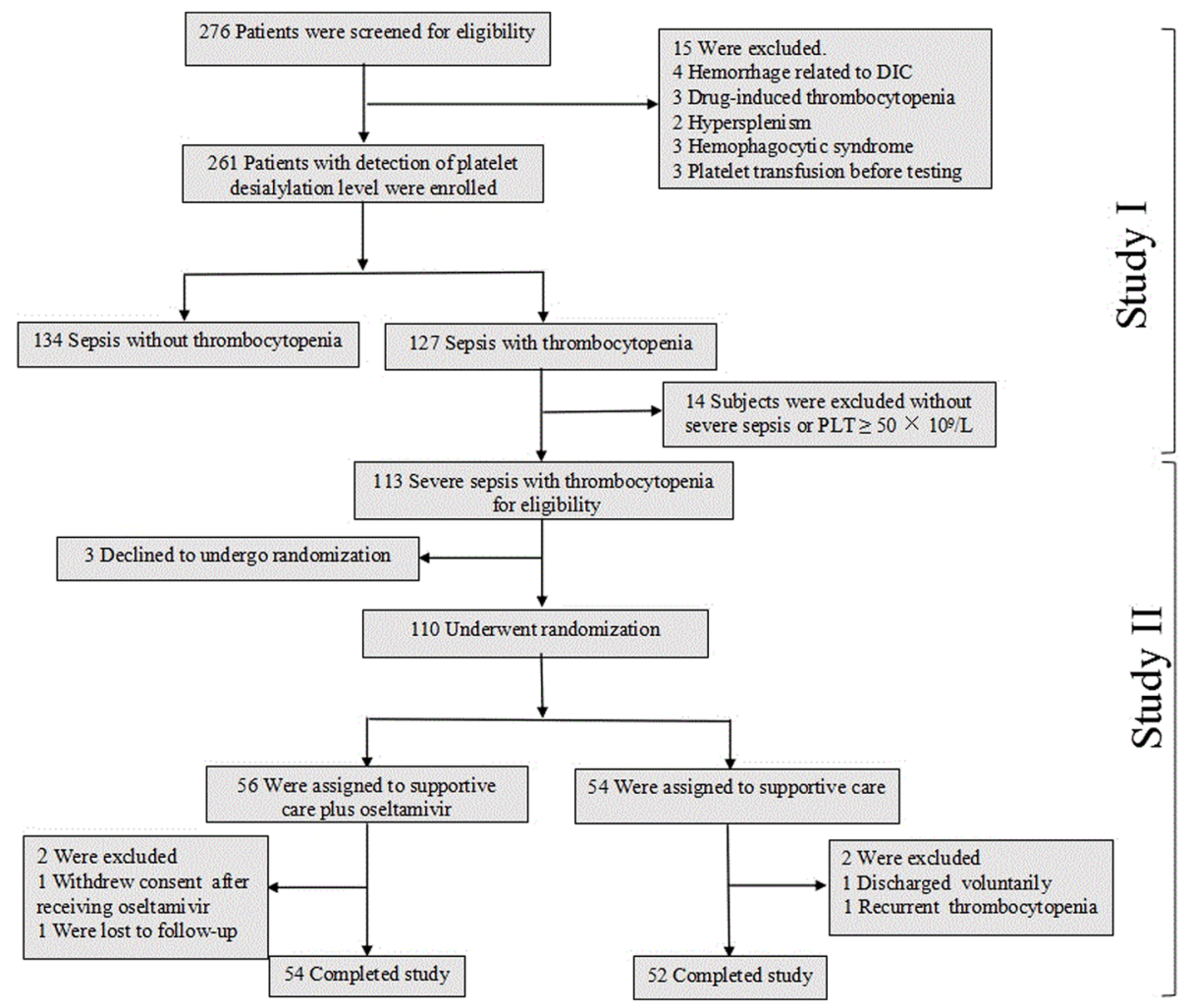

Fig. 1 Study flow chart. During the study period, 276 patients were screened for eligibility, and 261 patients met the inclusion criteria. Among them, 127 patients showed thrombocytopenia, of which 113 severe sepsis patients with platelet count less than $50 \times 10^{9} / \mathrm{L}$ were eligible for the subsequent randomized trial phase. Of them, 110 consented to participate in this phase and were randomly assigned to either receive antimicrobial therapy alone or to receive antimicrobial therapy plus oseltamivir

Both groups received appropriate antimicrobial agents and standard medical support based on the guidelines issued by the Surviving Sepsis Campaign [1]. The oseltamivir group additionally received five full days of oseltamivir therapy. The oseltamivir was administered orally or through a feeding tube at a dose of $75 \mathrm{mg}$ once every $12 \mathrm{~h}$. Time from randomization to the administration of oseltamivir was less than $24 \mathrm{~h}$. The antimicrobial agents were continuously administered until 3 days after the resolution of the physiological abnormalities related to the systemic inflammatory response syndrome (SIRS).

\section{Study population}

The studies were carried out in the Departments of Internal Medicine, and Medical ICUs at Yantai Yuhuangding Hospital, Yantaishan Hospital, Infectious Disease Hospital of Yantai, and Qilu Hospital, Shandong University, Shandong, China, from May 2014 to February 2016.

In study I, patients were eligible for enrollment if they were 18 to 80 years of age; if they presented with sepsis, severe sepsis, or septic shock; and if they had thrombocytopenia (platelet counts less than $100 \times 10^{9} / \mathrm{L}$ ) [35] at the time of admission. To analyze platelet desialylation levels, gender- and age-matched healthy adults were selected as normal controls. In study II, we only included the severe sepsis patients from study I whose platelet counts were less than $50 \times 10^{9} / \mathrm{L}$. In agreement with the study of the no inferiority test with a one-sided $5 \%$ significance level and a power of $80 \%$, a sample size of 50 patients per group was necessary, given an anticipated dropout rate of $10 \%$.

The diagnostic criteria for sepsis, severe sepsis, and septic shock were based on the Surviving Sepsis Campaign guidelines [1]. Thrombocytopenia was defined as a peripheral blood platelet count less than $100 \times 10^{9} / \mathrm{L}$ [35]. The exclusion criteria were patients who received chemotherapy or immunosuppressive agents within the prior 6 months, patients with disseminated intravascular coagulation, or drug (e.g., antibiotic or heparin)-associated thrombocytopenia, etc. The detailed eligibility and exclusion criteria are listed in Table 1.

The study was approved by the institutional ethics committee. Written informed consents were obtained from the study participants prior to inclusion in the study. For patients with coma or sedation, the consents were sought from their legal surrogates and were retrospectively gained from those who recovered mental capacity. This study was registered with the Chinese Clinical Trial Registry (ChiCTR; ChiCTR-IPR-16008542). 
Table 1 Eligibility and exclusion criteria of this study

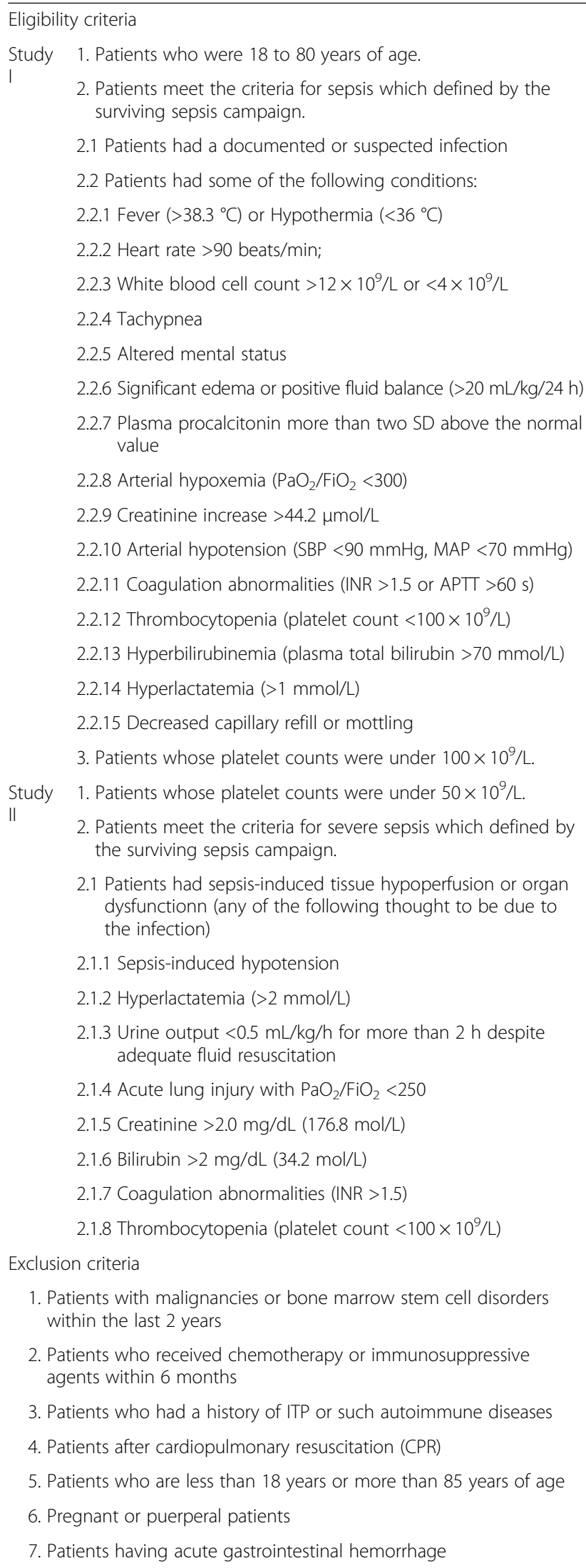

2.1 Patients had sepsis-induced tissue hypoperfusion or organ dysfunctionn (any of the following thought to be due to the infection)

2.1.1 Sepsis-induced hypotension

2.1.2 Hyperlactatemia ( $>2 \mathrm{mmol} / \mathrm{L}$ )

2.1.3 Urine output $<0.5 \mathrm{~mL} / \mathrm{kg} / \mathrm{h}$ for more than $2 \mathrm{~h}$ despite adequate fluid resuscitation

2.1.4 Acute lung injury with $\mathrm{PaO}_{2} / \mathrm{FiO}_{2}<250$

2.1.5 Creatinine $>2.0 \mathrm{mg} / \mathrm{dL}(176.8 \mathrm{~mol} / \mathrm{L})$

2.1.6 Bilirubin $>2 \mathrm{mg} / \mathrm{dL}(34.2 \mathrm{~mol} / \mathrm{L})$

2.1.7 Coagulation abnormalities (INR $>1.5)$

2.1.8 Thrombocytopenia (platelet count $<100 \times 10^{9} / \mathrm{L}$ )

\section{Exclusion criteria}

1. Patients with malignancies or bone marrow stem cell disorders within the last 2 years

2. Patients who received chemotherapy or immunosuppressive agents within 6 months

3. Patients who had a history of ITP or such autoimmune diseases

4. Patients after cardiopulmonary resuscitation (CPR)

5. Patients who are less than 18 years or more than 85 years of age

6. Pregnant or puerperal patients

7. Patients having acute gastrointestinal hemorrhage

Table 1 Eligibility and exclusion criteria of this study (Continued)

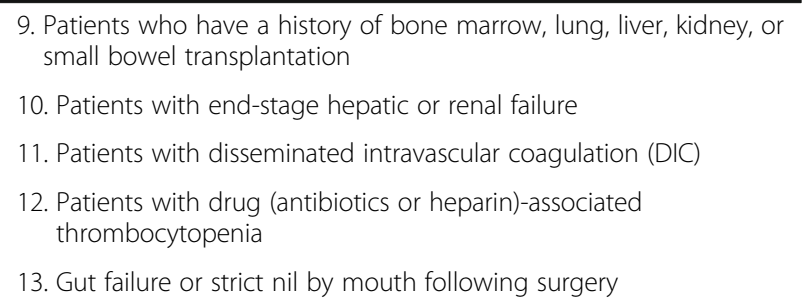

\section{Study end points}

The primary outcomes were platelet desialylation level at the entry of study I, overall platelet response rate within 14 days post-randomization, and all-cause mortality within 28 days post-randomization. Secondary outcomes included platelet recovery time, the occurrence of bleeding events, and the amount of platelets transfused within 14 days post-randomization.

Platelet response was defined as platelet counts returning to or above a normal level $\left(100 \times 10^{9} / \mathrm{L}\right)$.

Platelet recovery time was calculated as the date of randomization to the date when platelet counts were $>100 \times 10^{9} / \mathrm{L}$.

The criteria for platelet transfusion in patients with severe sepsis were documented as platelets less than $10 \times 10^{9} / \mathrm{L}$ without significant bleeding or platelets less than $20 \times 10^{9} / \mathrm{L}$ with significant bleeding risk [36].

\section{Data collection}

For each enrolled patient, the following variables were recorded: (1) general characteristics including age, gender, and primary diseases; (2) severity of illness as assessed by the Acute Physiology and Chronic Health Evaluation (APACHE) II and the Sequential Organ Failure Assessment (SOFA) scores; (3) platelet desialylation level; (4) laboratory data upon admission including hematologic and chemistry tests, blood coagulation assays, and arterial blood gas analysis; (5) screening tests for virus infection, serum galactomannan and $(1 \rightarrow 3)-\beta$-D-glucan assays, and bacterial culture from clinical specimens such as sputum, blood, urine, secretions, and drainage fluid; (6) interventions including mechanical ventilation and continuous renal replacement therapy (CRRT); (7) the development of complications such as acute respiratory distress syndrome (ARDS), DIC, and bleeding events; and (8) the amount of platelets transfused. Time from sepsis onset for measurement of all laboratory parameters was limited within $24 \mathrm{~h}$.

\section{Assessment of platelet desialylation}

Ethylenediaminetetraacetic acid anti-coagulated whole blood was obtained from sepsis patients and healthy controls by venipuncture. Platelet-rich plasma was isolated by centrifugation at $200 \mathrm{~g}$ for $10 \mathrm{~min}$. Fresh platelets were separated from platelet-rich plasma by centrifugation at 
$850 \mathrm{~g}$ for $5 \mathrm{~min}$, washed in buffer A $(140 \mathrm{mM} \mathrm{NaCl}, 5 \mathrm{mM}$ $\mathrm{KCl}, 12 \mathrm{mM}$ trisodium citrate, $10 \mathrm{mM}$ glucose, $12.5 \mathrm{mM}$ sucrose, $1 \mu \mathrm{g} / \mathrm{mL}$ prostaglandin $\mathrm{E} 1, \mathrm{pH} 6.0$ ), and resuspended in buffer B (10 mM HEPES, $140 \mathrm{mM} \mathrm{NaCl}, 3 \mathrm{mM}$ $\mathrm{KCl}, 0.5 \mathrm{mM} \mathrm{MgCl}, 10 \mathrm{mM}$ glucose, and $0.5 \mathrm{mM}$ $\mathrm{NaHCO}_{3}, \mathrm{pH}$ 7.4).

To detect platelet desialylation, PE-Cy5-labeled antihuman CD41 monoclonal antibodies $(20 \mu \mathrm{L}$ per test, $4 \mu \mathrm{g} / \mathrm{mL}$; BD Bioscience, San Jose, CA, USA) were used to label human platelets. FITC-labeled Ricinus communis agglutinin I (RCA-I, $5 \mu \mathrm{g} / \mathrm{mL}$; Vector Laboratories, Burlingame, CA, USA), Erythrina cristagalli lectin (ECL, $10 \mu \mathrm{g} / \mathrm{mL}$; EY Laboratories, San Mateo, CA, USA), and Succinyl Triticum vulgare lectin (sWGA, $0.1 \mu \mathrm{g} / \mathrm{mL}$; EY Laboratories) were used to analyze sialic acid exposure on platelet surfaces. Platelets $\left(1 \times 10^{6}\right)$ were incubated with anti-CD41 and RCA-I, ECL, or sWGA, respectively for $20 \mathrm{~min}$, washed twice, and resuspended. The percentages of platelets positive for RCA-I, ECL, or sWGA represented the levels of platelet desialylation [31].

\section{Statistics}

Data analysis was performed using the SPSS 16.0 statistical software package (SPSS Inc., Chicago, IL, USA). Normally distributed variables were expressed as the mean \pm standard deviation (SD), while skewed variables were expressed as the median (interquartile range). Between-group comparisons were evaluated by the twotailed unpaired Student's $t$ test (for normally distributed data) and Mann-Whitney $U$ test (for skewed data). The comparison of categorical variables was processed by the chi-squared $\left(\chi^{2}\right)$ test. Multiple stepwise regression analysis was used to assess the association between platelet count and the other variables, while independent variables correlating with the 28-day mortality were determined by a multiple logistic regression analysis. Survival analysis was conducted based on whether oseltamivir was used, and differences in survival rates between groups were compared using the log-rank test. A multivariate analysis with the Cox proportional hazards regression model was used to assess the influence of each variable on the response to oseltamivir treatment. A $p$ value less than 0.05 was considered statistically significant.

\section{Results}

Baseline characteristics and clinical features for all of the patients

During the study period, 276 patients were admitted, and 261 patients met the criteria of study I (Fig. 1). Among them, 134 were septic patients without thrombocytopenia, and 127 were patients with thrombocytopenia. Additional file 1: Table S2 shows the characteristics of the 261 patients upon admission.
Among the 127 septic patients with thrombocytopenia, 110 severe sepsis patients whose platelet counts were less than $50 \times 10^{9} / \mathrm{L}$ were enrolled in study II. After randomization, 52 patients were treated with antibiotics alone (the control group), whereas the other 54 patients were treated with antibiotics plus oseltamivir (the oseltamivir group). The 28-day follow-up was completed in $96.4 \%$ of the patients (Fig. 1).

\section{Comparison of septic patients with or without thrombocytopenia}

Table 2 details the demographic data of septic patients with or without thrombocytopenia. Patients with thrombocytopenia had higher SOFA scores $[10(5,12)$ vs $5(3.25,7), P<0.001]$, PCT levels $[6.69(1.0,38.9)$ vs 1.55 (0.32, 5.36), $P<0.001$ ], and creatinine levels [88 (63.3, $225)$ vs $81(56,119.0) \mu \mathrm{mol} / \mathrm{L}, P=0.036]$ but had a lower mean arterial pressure $(79.6 \pm 14.7$ vs $86.8 \pm 15.5 \mathrm{mmHg}$, $P<0.001)$ than patients without thrombocytopenia. Patients with thrombocytopenia had a higher occurrence of renal replacement $(25.2$ vs $14.2 \%, P=0.029)$ and septic shock ( 18.9 vs $8.2 \%, P=0.017)$ than patients without thrombocytopenia. The 28-day mortality rate was higher in patients with thrombocytopenia than in patients without thrombocytopenia (36.2 vs $14.2 \%$, respectively; $P<0.001)$. And there were significant differences between patients with and without thrombocytopenia in the incidence rate of bloodstream infection, lung infection, and the infection pathogens of Escherichia coli, New Bunya virus, Pseudomonas aeruginosa, and Staphylococcus aureus. Multiple stepwise regression analysis of the association of thrombocytopenia and the clinical parameters with significant differences in Table 2 revealed that the bloodstream infection and the infection pathogen of New Bunya virus were independent risk factors for thrombocytopenia in sepsis patients. In contrast, the infection caused by $P$. aeruginosa and $S$. aureus were negatively associated with thrombocytopenia (Additional file 1: Table S2). Multivariate logistic regression analyses were performed to determine if there were any associations between risk factors and 28-day mortality in the 261 patients, the risk factors including age, gender, SOFA, platelet count, mechanical ventilation, continuous renal replacement therapy (CRRT), acute respiratory distress syndrome (ARDS), and septic shock (Additional file 1: Table S3). It was demonstrated that platelet count was an independent factor negatively associated with the 28-day mortality rate $(\mathrm{OR}=0.963,95 \%$ CI $0.930-0.997, P=0.033$ ), which was consistent with other reports [3]. SOFA was also an independent risk factor for 28-day mortality rate $(\mathrm{OR}=1.808,95 \% \mathrm{CI}$ 1.246-2.623, $P=0.002$ ).

To compare the platelet desialylation levels between septic patients with or without thrombocytopenia, we quantified the binding of fluorescein-conjugated lectins, 
Table 2 Comparison between the sepsis patients with and without thrombocytopenia

\begin{tabular}{|c|c|c|c|}
\hline Variables & Sepsis without thrombocytopenia $(n=134)$ & Sepsis with thrombocytopenia $(n=127)$ & $P$ value \\
\hline Age (year) & $69.5(54.5,78.3)$ & $68(55.0,77.0)$ & 0.468 \\
\hline Male & $91(67.9)$ & $84(66.1)$ & 0.793 \\
\hline APACHE ॥ & $18.5(15.3,23.8)$ & $20(10,24.8)$ & 0.861 \\
\hline Predicted death rate (\%) & $31.5(21,45.2)$ & $37.3(20.4,53.5)$ & 0.217 \\
\hline SOFA & $5(3.25,7)$ & $10(5,12)$ & $<0.001$ \\
\hline $\mathrm{PCT}(\mathrm{ng} / \mathrm{mL})$ & $1.55(0.32,5.36)$ & $6.69(1.0,38.9)$ & $<0.001$ \\
\hline $\mathrm{SA}(\mathrm{mmol} / \mathrm{L})$ & $732.8 \pm 156.5$ & $669.1 \pm 143.9$ & 0.089 \\
\hline MAP $(\mathrm{mmHg})$ & $86.8 \pm 15.5$ & $79.6 \pm 14.7$ & $<0.001$ \\
\hline Heart rate (beat/min) & $93.3(88.0,106.3)$ & $98(84,116)$ & 0.221 \\
\hline White blood cell $\left(\times 10^{9} / \mathrm{L}\right)$ & $12.5(8.4,15.4)$ & $14.1(7.81,15.5)$ & 0.352 \\
\hline Platelet count $\left(\times 10^{9} / \mathrm{L}\right)$ & $196.5(143.5,299.75)$ & $45(33.25,64.75)$ & $<0.001$ \\
\hline BUN (mmol/L) & $7.4(5.18,17.3)$ & $7.51(4.06,14.6)$ & 0.402 \\
\hline Creatinine $(\mu \mathrm{mol} / \mathrm{L})$ & $81(56.0,119.0)$ & $88(63.3,225.0)$ & 0.036 \\
\hline Total bilirubin $(\mu \mathrm{mol} / \mathrm{L})$ & $14.8(9.9,23.1)$ & $18.8(11.4,35.0)$ & 0.098 \\
\hline Albumin (g/L) & $25.5 \pm 3.48$ & $24.8 \pm 5.75$ & 0.267 \\
\hline \multicolumn{4}{|l|}{ Infection pathogens (\%) } \\
\hline Escherichia coli & $12(8.96)$ & $25(19.7)$ & 0.020 \\
\hline Klebsiella pneumoniae & $22(16.4)$ & $11(8.66)$ & 0.065 \\
\hline Pseudomonas aeruginosa & $30(22.4)$ & $11(8.66)$ & 0.030 \\
\hline Acinetobacter baumanii & $13(9.70)$ & $7(5.51)$ & 0.248 \\
\hline Staphylococcus aureus & $21(15.7)$ & $5(3.93)$ & 0.020 \\
\hline Streptococcus pneumonae & $1(0.75)$ & $2(1.57)$ & 0.641 \\
\hline Enterococcus faecium & $5(3.73)$ & $4(3.15)$ & 0.999 \\
\hline Chicken enterococcus & $1(0.75)$ & $2(1.57)$ & 0.614 \\
\hline Proteus mirabilis & 0 & $1(0.78)$ & 0.487 \\
\hline Candida albicans & $1(0.75)$ & $2(1.57)$ & 0.614 \\
\hline Aspergillus & $3(2.24)$ & $7(5.51)$ & 0.207 \\
\hline Viruses & $17(12.7)$ & $36(28.3)$ & 0.002 \\
\hline New Bunya virus & 0 & $21(16.5)$ & $<0.001$ \\
\hline Influenza A virus subtype H1N1 & $6(4.48)$ & $6(4.72)$ & 0.999 \\
\hline Others & $11(10.4)$ & $9(9.45)$ & 0.818 \\
\hline \multicolumn{4}{|l|}{ Infection sites (\%) } \\
\hline Lung & $69(51.5)$ & $29(22.8)$ & $<0.001$ \\
\hline Bloodstream & $8(5.97)$ & $33(23.6)$ & $<0.001$ \\
\hline Abdominal cavity & $11(8.21)$ & $15(11.1)$ & 0.409 \\
\hline Hepatobiliary system & $8(5.97)$ & $9(6.9)$ & 0.804 \\
\hline Urinary system & $6(4.48)$ & $8(6.9)$ & 0.589 \\
\hline Surgical incision & $3(2.24)$ & $3(6.9)$ & 0.632 \\
\hline Skin and soft tissue & $2(1.49)$ & $2(1.4)$ & 0.668 \\
\hline Central nervous system & $2(1.49)$ & $1(1.4)$ & 0.520 \\
\hline Others & $25(18.7)$ & $27(21.3)$ & 0.644 \\
\hline \multicolumn{4}{|l|}{ Platelet desialylation level (\%) } \\
\hline RCA-I & $90.14 \pm 8.88$ & $95.64 \pm 4.19$ & 0.014 \\
\hline ECL & $2.33 \pm 1.90$ & $5.70 \pm 4.37$ & $<0.001$ \\
\hline
\end{tabular}


Table 2 Comparison between the sepsis patients with and without thrombocytopenia (Continued)

\begin{tabular}{llll}
\hline sWGA & $0.15 \pm 0.95$ & $0.50 \pm 0.293$ & $<0.001$ \\
Mechanical ventilation & $63(47.0)$ & $72(56.7)$ & 0.137 \\
Renal replacement therapy & $19(14.2)$ & $32(25.2)$ & 0.029 \\
Septic shock & $11(8.2)$ & $24(18.9)$ & 0.017 \\
ARDS & $30(22.4)$ & $34(26.8)$ & 0.472 \\
Bleeding & $32(23.9)$ & $29(22.8)$ & 0.884 \\
28-day mortality & $19(14.2)$ & $46(36.2)$ & $<0.001$ \\
\hline
\end{tabular}

Data are presented as number (percentage) or median (interquartile range); APACHE II acute physiology and chronic health evaluation scoring system, SOFA sequential organ failure assessment, $P C T$ procalcitonin, $A R D S$ acute respiratory distress syndrome, MAP mean arterial pressure, SA sialic acid, RCA-I Ricinus communis agglutinin I, ECL Erythrina cristagalli lectin, sWGA Succinyl Triticum vulgare lectin, BUN blood urea nitrogen

which target exposed sialic acids following GP desialylation as follows: ECL, which has a specificity toward galactose residues and the highest binding activity toward galactosyl $(\beta-1,4) N$-acetylglucosamine; RCA-I, which binds preferentially to oligosaccharides ending in galactose but may also interact with $N$-acetylgalactosamine; and sWGA, which has a specificity toward $\mathrm{N}$-acetylglucosamine. The data demonstrated that platelet desialylation increased significantly in septic patients with thrombocytopenia compared to those without thrombocytopenia, as detected with ECL, RCA-I, or sWGA lectins (Additional file 1: Figure S1).

\section{Comparison of antimicrobial therapy plus oseltamivir with antimicrobial therapy alone in severe sepsis patients with thrombocytopenia}

The baseline demographic data of patients treated with antibiotics plus oseltamivir (oseltamivir group) or with antibiotics alone (control group) are listed in Additional file 1: Table S4. There were no differences between the two study groups (at a significance level of less than 0.05 ) with respect to any demographic variable, showing a comparability of our data. There was no difference in adequate antibiotic treatment between groups. The duration of the antimicrobial treatment was not different in the oseltamivir group compared with the control group $[11(9,13.25)$ vs $10(9,13) ; P=0.418]$. No significant between-group differences were found with respect to the sites of infection or the infectious pathogens. All of the laboratory tests presented no significant differences between the two groups, except for some isolated indices.

Table 3 demonstrates the responses and outcomes of patients in the oseltamivir group and the control group. Within 15 days after randomization, platelet response was achieved in 45 of the 54 patients in the oseltamivir group (83.3\%) compared with 34 of the 52 patients in the control group $(65.4 \% ; P=0.045)$. In patients who had a platelet response, the platelet recovery time was 5 days with quartiles of 4-6 days in the oseltamivir group compared with 7 days with quartiles of 5-10 days in the control group $(P=0.003)$. Additional file 1: Figure S2 displays the changes in the platelet counts of the oseltamivir and control groups over time. At baseline, the platelet counts were $41.81 \pm 10.29 \times 10^{9} / \mathrm{L}$ in the oseltamivir group and $46.69 \pm 9.13 \times 10^{9} / \mathrm{L}$ in the control group, with no significant difference between these two groups $(P=0.560)$. Platelet counts in both groups increased over time and reached $91.30 \pm 21.51 \times 10^{9} / \mathrm{L}$ in the oseltamivir group and $58.17 \pm 32.99 \times 10^{9} / \mathrm{L}$ in the control group on the fourth day after the initiation of oseltamivir treatment $(P=0.031)$. From day 4 through day 9 , the difference continued to be statistically significant between the groups, and on the 10th day, platelet counts were $198.8 \pm 82.6 \times 10^{9} / \mathrm{L}$ in the oseltamivir group and $147.7 \pm 81.78 \times 10^{9} / \mathrm{L}$ in the control group $(P=0.684)$. The amount of platelets transfused decreased significantly in the oseltamivir group $(0.676 \pm 1.36$ apheresis units) compared to the control group $(1.35 \pm 1.98$ apheresis units; $P=0.044$; Table 3). The data indicated that oseltamivir could shorten the platelet recovery time and reduce platelet transfusion quantity. No special adverse events during the oseltamivir therapy were observed (Additional file 1: Table S5). The platelet desialylation levels were analyzed in 25 patients after oseltamivir therapy. The sWGA FITC-labeled lectins declined significantly compared to those before treatment. However, neither RCA-I nor ECL FITC-labeled lectins showed significant change after oseltamivir treatment (Additional file 1: Figure S4).

The overall 28-day mortality rate in the two groups combined was $38.7 \%$, with $35.2 \%$ in the oseltamivir group and $42.3 \%$ in the control group $(P=0.55$; Table 3$)$.

Table 3 Responses and outcomes of patients in the oseltamivir group and control group

\begin{tabular}{llll}
\hline Variables & $\begin{array}{l}\text { Control } \\
(n=52)\end{array}$ & $\begin{array}{l}\text { Oseltamivir } \\
(n=54)\end{array}$ & $P$ value \\
\hline Platelet response rate, \% (n) & $65.4(34)$ & $83.3(45)$ & 0.045 \\
Platelet recovery time (day) & $7(5,10)$ & $5(4,6)$ & 0.003 \\
Platelet transfusion (apheresis unit) & $1.35 \pm 1.98$ & $0.676 \pm 1.36$ & 0.044 \\
Bleeding (\%) & $9(17.3)$ & $11(20.4)$ & 0.805 \\
28-day mortality (\%) & $22(42.3)$ & $19(35.2)$ & 0.550 \\
\hline
\end{tabular}


There was no difference in survival over time based on whether oseltamivir was used (Additional file 1: Figure S3). Multivariate Cox proportional hazards models were used to estimate the variables that were associated with the responses in the oseltamivir and control groups, respectively, including age, sex, APACHE II score, SOFA score, platelet recovery time, and platelet count. It was revealed that the SOFA score and platelet recovery time were independent indicators of oseltamivir therapy (Table 4). The main reason for all of the mortalities was multiple-organ failure.

Oseltamivir treatment was well-tolerated in our study. No patient reported side effects that were severe enough to necessitate the discontinuation of treatment. Eighteen patients with the digestive tract and respiratory diseases or both tolerated the medication well (Additional file 1: Table S5). On the whole, the incidence of adverse events in oseltamivir group was similar to the control group.

\section{Discussion}

According to the 2016 international consensus definitions for sepsis and septic shock (Sepsis-3), sepsis is defined as life-threatening organ dysfunction caused by a dysregulated host response to an infection. Organ dysfunction can be identified as an acute change in the total SOFA score $\geq 2$ consequent to the infection. The new definition of sepsis reflects an up-to-date view of pathobiology, particularly in regards to what distinguishes sepsis from uncomplicated infection [37]. Although our study was initiated in 2014, at which point the diagnostic criteria for sepsis was based on the Surviving Sepsis Campaign guidelines (Sepsis-2), 253 of the 261 sepsis patients included had SOFA scores $\geq 2$. Thus, even by the more "strict" criteria of Sepsis-3, most patients had sepsis involving organ dysfunction rather than an infection plus an accompanying inflammatory response alone.

In this study, we demonstrated that the platelet desialylation increased significantly in septic patients with thrombocytopenia compared to those without

Table 4 Multivariate Cox proportional hazard analysis of oseltamivir therapy

\begin{tabular}{lcccc}
\hline Variables & $\begin{array}{l}\text { Hazard } \\
\text { ratio }\end{array}$ & \multicolumn{2}{l}{$95 \% \mathrm{Cl}$} & $P$ \\
\cline { 3 - 4 } & & Lower & Upper & \\
\hline Age & 1.020 & 0.990 & 1.051 & 0.189 \\
Sex & 0.938 & 0.726 & 1.212 & 0.626 \\
APACHE II & 1.105 & 0.951 & 1.284 & 0.191 \\
SOFA & 0.578 & 0.365 & 0.965 & 0.029 \\
Platelet recovery time & 0.798 & 0.636 & 0.990 & 0.042 \\
Platelet count & 1.011 & 0.983 & 1.039 & 0.463 \\
\hline
\end{tabular}

$95 \% \mathrm{Cl}$ indicates $95 \%$ confidence interval. APACHE II and SOFA score were obtained at patients' admission. APACHE II acute physiology and chronic health evaluation scoring system, SOFA sequential organ failure assessment thrombocytopenia as detected with ECL, RCA-I, or sWGA lectins, indicating that thrombocytopenia was associated with increased platelet desialylation in septic patients. This research confirms the desialylation pathogenesis of sepsis thrombocytopenia and provides new countermeasures for the clinical treatment of thrombocytopenia in sepsis patients.

Greenberg et al. pretreated platelets with neuraminidases in vitro and found that removal of $15 \%$ of platelet sialic acid led to complete platelet clearance within $1 \mathrm{~h}$ after injection back to the circulation [38]. Moreover, an exponential correlation was showed between the shortening of platelet life span in the circulation and the content of sialic acid removed by neuraminidases in vitro [39]. In addition, direct injection of neuraminidases to mammals markedly accelerated the clearance and shorted the life span of platelets $[40,41]$. These studies suggest that neuraminidases can significantly affect platelet life span by causing platelet desialylation.

Next, we performed a randomized controlled study in which the severe sepsis patients who had thrombocytopenia with platelet counts less than $50 \times 10^{9} / \mathrm{L}$ were randomly assigned to receive antimicrobial therapy alone or antimicrobial therapy plus oseltamivir. The data demonstrated that the addition of oseltamivir to antibiotic therapy could significantly increase the platelet response rate, shorten the platelet recovery time, and reduce platelet transfusion. The oseltamivir was administered for 5 days according to the drug instruction, yet its effect appeared to persist well beyond discontinuation of the drug and the difference continued to be statistically significant between the oseltamivir and control group.

It is not clear whether correcting the platelet count will result in a decrease in mortality. In this study, the multivariate analysis by Cox proportional hazards models showed that the SOFA score and platelet recovery time were independent indicators of oseltamivir therapy. Therefore, the main cause for all of the cases of mortality was multiple-organ failure [42]. This result might explain why there was no difference in the overall 28-day mortality rate or in the survival over time based on whether oseltamivir was used in this study.

However, in critical care medicine, it is commonly agreeable that when evaluating an intervention in critically ill patients, it is not necessarily associated with improved mortality. In a study which systematically reviewed the available literature to identify multicenter RCTs assessing mortality as a primary outcome in ICU patients, it is demonstrated that relatively few of the RCTs conducted in ICUs and using mortality as a primary outcome show a beneficial impact of the intervention on the survival of critically ill patients [43]. Many other studies also stimulate research on end points other than crude differences in mortality rates [44]. 
One limitation of our trial is its open-label nature. Given that most severe sepsis patients with thrombocytopenia in study II are acute and/or critically ill patients with multiple-organ failure, a blinded study do not seem possible. Moreover, all end points in our study were based on objectively measured laboratory values rather than clinical impressions or assessments of symptoms which would be impacted by a clinician's subjective bias during data collection and evaluation of study parameters. So a placebo with blinding was not included in the study design.

Septic patients with thrombocytopenia carry particularly poor prognosis and often require platelet transfusions. Platelets are a limited resource and have potential for causing transfusion reactions. Beyond the role in hemostasis and thrombosis, platelets modulate inflammatory reactions and immune responses [45]. The study showed that despite the absence of a difference in 28-day mortality, oseltamivir shortened the recovery time from thrombocytopenia, reduced the need for platelet transfusion, and saved the cost. Therefore, a medication with a relatively good safety profile which is readily available and can decrease the requirements for platelet transfusions would be beneficial. Additionally, early recovery from thrombocytopenia helps to prevent the coagulopathy and enhance the immunity. Our study confirmed that desialylation pathogenesis of sepsis thrombocytopenia provides new strategy for the clinical treatment of thrombocytopenia in sepsis patients.

\section{Conclusions}

Thrombocytopenia was associated with increased platelet desialylation in septic patients. The addition of oseltamivir could significantly increase the platelet response rate, shorten the platelet recovery time and reduce platelet transfusion.

\section{Additional files}

Additional file 1: Figure S1. Flow cytometric analysis of $\beta$-galactose or $\beta$-GICNAC exposure on platelet glycoproteins. Figure S2. Changes in platelet counts of the oseltamivir and control groups over time. Figure S3. Survival curves of thrombocytopenia patients, according to treatment group. Figure S4. Platelet desialylation levels before and after the oseltamivir treatment. Table S1. Baseline characteristics of the all patients $(n=261)$. Table S2. Factors affecting the thrombocytopenia in the multiple stepwise regression model $(n=261)$. Table S3. Multivariate analysis of the effects of various clinical parameters at study entry on the odds ratio for 28-day mortality $(n=261)$. Table S4. Baseline demographic data of patients treated with antibiotics plus oseltamivir (oseltamivir group) or with antibiotics alone (control group). Table S5. The adverse events in oseltamivir treatment group and control group in study II $(n=106)$. (DOC $1586 \mathrm{~kb})$

\section{Acknowledgements}

We thank the critical care nurses of Yantai Yuhuangding Hospital Affiliated to Qingdao University, who collected much of the data during the study.

\section{Funding}

This work was supported by grants from the Major Research plan of the National Natural Science Foundation of China (No. 91442204), National Natural
Science Foundation for Distinguished Young Scholars of China (No. 81125002), National Natural Science Foundation of China (No. 81370623, No. 81270578, No. 81470284), State Program of National Natural Science Foundation of China for Innovative Research Group (81321061), State Key Clinical Specialty of China for Blood Disorders, Tai Shan Scholar Foundation, and Yantai Municipal Science and Technology Commission (No. 2014WS012).

Availability of data and materials

The datasets supporting the conclusions of this article are included within the article and its additional file.

\section{Authors' contributions}

$J P$ and $M H$ were primary investigators. JP, LyL and MfL wrote the manuscript draft. MfL, XIL, YyY, XL, JG, KIF, SyG, YfL, LH, JhQ, XhT, WtW, XIZ, QxY, YfZ, LnW, MH, LyL and JP collected data. MfL performed statistical analysis and interpretation. All authors read and approved the final manuscript.

\section{Competing interests}

The authors declare that they have no competing interests.

\section{Consent for publication}

Not applicable.

\section{Ethics approval and consent to participate}

The study was approved by the Medical Ethics Committee of Qilu Hospital, Shandong University, Yantai Yuhuangding Hospital, Yantaishan Hospital, and Infectious Disease Hospital of Yantai. Written informed consent was obtained from the study participants before enrollment in accordance with the Declaration of Helsinki. For patients with coma or sedation, the consents were sought from their legal surrogates and were retrospectively gained from those who recovered mental capacity.

\section{Publisher's Note}

Springer Nature remains neutral with regard to jurisdictional claims in published maps and institutional affiliations.

\section{Author details}

'Department of Hematology, Qilu Hospital, Shandong University, Jinan 250012, China. ${ }^{2}$ Intensive Care Unit, and Clinical Laboratory, Yantai Yuhuangding Hospital Affiliated to Qingdao University, Yantai 264000, Shandong, China. ${ }^{3}$ Department of Emergency, Affiliated Hospital of Shandong University of Traditional Chinese Medicine, Jinan, China. ${ }^{4}$ Division of Preventive Medicine, Center for Disease Control and Prevention of Yantai Development Zone, Yantai, China. ${ }^{5}$ Department of Internal Medicine, Infectious Disease Hospital of Yantai, Yantai, China. ${ }^{6}$ Intensive Care Unit, Yantaishan Hospital of Yantai, Yantai, China. ${ }^{7}$ Shandong Provincial Key Laboratory of Immunohematology, Qilu Hospital, Shandong University, Jinan, China.

Received: 21 March 2017 Accepted: 2 May 2017

Published online: 11 May 2017

\section{References}

1. Dellinger RP, Levy MM, Rhodes A, et al. Surviving Sepsis Campaign: international guidelines for management of severe sepsis and septic shock, 2012. Intensive Care Med. 2013;39:165-228.

2. Angus DC, van der Poll T. Severe sepsis and septic shock. N Engl J Med. 2013;369:2063.

3. Lim SY, Jeon E, Kim HJ, et al. The incidence, causes, and prognostic significance of new-onset thrombocytopenia in intensive care units: a prospective cohort study in a Korean hospital. J Korean Med Sci. 2012;27:1418-23.

4. Brogly N, Devos P, Boussekey N, et al. Impact of thrombocytopenia on outcome of patients admitted to ICU for severe community-acquired pneumonia. J Infect. 2007;55:136-40.

5. Moreau D, Timsit JF, Vesin A, et al. Platelet count decline: an early prognostic marker in critically ill patients with prolonged ICU stays. Chest. 2007:131:1735-41.

6. Sharma B, Sharma M, Majumder M, et al. Thrombocytopenia in septic shock patients - a prospective observational study of incidence, risk factors and correlation with clinical outcome. Anaesth Intensive Care. 2007;35:874-80. 
7. Vanderschueren S, De Weerdt A, Malbrain M, et al. Thrombocytopenia and prognosis in intensive care. Crit Care Med. 2000;28:1871-6.

8. de Stoppelaar SF, van't Veer $C$, van der Poll T. The role of platelets in sepsis. Thromb Haemost. 2014;112:666-77.

9. Cines DB, Bussel JB, Liebman HA, et al. The ITP syndrome: pathogenic and clinical diversity. Blood. 2009;113:6511-21.

10. Neame PB, Kelton JG, Walker IR, et al. Thrombocytopenia in septicemia: the role of disseminated intravascular coagulation. Blood. 1980;56:88-92.

11. Andonegui $G$, Kerfoot SM, McNagny K, et al. Platelets express functional Toll-like receptor-4. Blood. 2005;106:2417-23.

12. Wilson JJ, Neame PB, Kelton JG. Infection-induced thrombocytopenia. Semin Thromb Hemost. 1982:8:217-33.

13. Francois B, Trimoreau F, Vignon $\mathrm{P}$, et al. Thrombocytopenia in the sepsis syndrome: role of hemophagocytosis and macrophage colony-stimulating factor. Am J Med. 1997;103:114-20.

14. Xiang B, Zhang G, Guo L, et al. Platelets protect from septic shock by inhibiting macrophage-dependent inflammation via the cyclooxygenase 1 signalling pathway. Nat Commun. 2013;4:2657

15. Yin H, Stojanovic-Terpo A, Xu W, et al. Role for platelet glycoprotein Ib-IX and effects of its inhibition in endotoxemia-induced thrombosis, thrombocytopenia, and mortality. Arterioscler Thromb Vasc Biol. 2013;33:2529-37.

16. Pockros PJ, Duchini A, McMillan R, et al. Immune thrombocytopenic purpura in patients with chronic hepatitis C virus infection. Am J Gastroenterol. 2002;97:2040-5.

17. Torre D, Pugliese A. Platelets and HIV-1 infection: old and new aspects. Curr HIV Res. 2008;6:411-8

18. Tavil B, Unal S, Aytac-Elmas S, et al. Weekly long-term intravenous immunoglobulin for refractory parvovirus B19 and Epstein-Barr virusinduced immune thrombocytopenic purpura. Turk J Pediatr. 2008;50:74-7.

19. Grewal PK, Aziz PV, Uchiyama S, et al. Inducing host protection in pneumococcal sepsis by preactivation of the Ashwell-Morell receptor. Proc Natl Acad Sci U S A. 2013;110:20218-23.

20. Grewal PK, Uchiyama S, Ditto D, et al. The Ashwell receptor mitigates the lethal coagulopathy of sepsis. Nat Med. 2008;14:648-55.

21. Chen X, Varki A. Advances in the biology and chemistry of sialic acids. ACS Chem Biol. 2010;5:163-76.

22. Roggentin $P$, Schauer $R$, Hoyer $L L$, et al. The sialidase superfamily and its spread by horizontal gene transfer. Mol Microbiol. 1993;9:915-21.

23. Monti E, Bonten E, D'Azzo A, et al. Sialidases in vertebrates: a family of enzymes tailored for several cell functions. Adv Carbohydr Chem Biochem. 2010;64:403-79.

24. Tribulatti MV, Mucci J, Van Rooijen N, et al. The trans-sialidase from Trypanosoma cruzi induces thrombocytopenia during acute Chagas' disease by reducing the platelet sialic acid contents. Infect Immun. 2005;73:201-7.

25. King SJ, Hippe KR, Weiser JN. Deglycosylation of human glycoconjugates by the sequential activities of exoglycosidases expressed by Streptococcus pneumoniae. Mol Microbiol. 2006;59:961-74.

26. Manco S, Hernon F, Yesilkaya H, et al. Pneumococcal neuraminidases A and $B$ both have essential roles during infection of the respiratory tract and sepsis. Infect Immun. 2006;74:4014-20.

27. Sorensen AL, Rumjantseva $V$, Nayeb-Hashemi S, et al. Role of sialic acid for platelet life span: exposure of beta-galactose results in the rapid clearance of platelets from the circulation by asialoglycoprotein receptor-expressing liver macrophages and hepatocytes. Blood. 2009;114:1645-54.

28. Hoffmeister KM, Felbinger TW, Falet $\mathrm{H}$, et al. The clearance mechanism of chilled blood platelets. Cell. 2003;112:87-97.

29. Hoffmeister KM, Josefsson EC, Isaac NA, et al. Glycosylation restores survival of chilled blood platelets. Science. 2003;301:1531-4.

30. Jansen AJ, Peng J, Zhao HG, et al. Sialidase inhibition to increase platelet counts: a new treatment option for thrombocytopenia. Am J Hematol. 2015;90:E94-95.

31. Jansen AJ, Josefsson EC, Rumjantseva $\mathrm{V}$, et al. Desialylation accelerates platelet clearance after refrigeration and initiates GPIbalpha metalloproteinase-mediated cleavage in mice. Blood. 2012;119:1263-73.

32. Hata K, Koseki K, Yamaguchi K, et al. Limited inhibitory effects of oseltamivir and zanamivir on human sialidases. Antimicrob Agents Chemother. 2008:52:3484-91.

33. Moore ML, Chi MH, Zhou W, et al. Cutting edge: oseltamivir decreases T cell GM1 expression and inhibits clearance of respiratory syncytial virus: potential role of endogenous sialidase in antiviral immunity. J Immunol. 2007;178:2651-4.
34. Shao L, Wu Y, Zhou H, et al. Successful treatment with oseltamivir phosphate in a patient with chronic immune thrombocytopenia positive for anti-GPIb/IX autoantibody. Platelets. 2015;26:495-7.

35. Thiolliere F, Serre-Sapin AF, Reignier J, et al. Epidemiology and outcome of thrombocytopenic patients in the intensive care unit: results of a prospective multicenter study. Intensive Care Med. 2013;39:1460-8.

36. Van der Linden T, Souweine B, Dupic L, et al. Management of thrombocytopenia in the ICU (pregnancy excluded). Ann Intensive Care. 2012;2:42.

37. Seymour CW, Liu VX, Iwashyna TJ, et al. Assessment of clinical criteria for sepsis: for the third international consensus definitions for sepsis and septic shock (Sepsis-3). JAMA. 2016;315:762-74.

38. Greenberg J, Packham MA, Cazenave JP, et al. Effects on platelet function of removal of platelet sialic acid by neuraminidase. Lab Invest. 1975;32:476-84.

39. Kotze HF, van Wyk V, Badenhorst PN, et al. Influence of platelet membrane sialic acid and platelet-associated lgG on ageing and sequestration of blood platelets in baboons. Thromb Haemost. 1993;70:676-80.

40. Choi SI, Simone JV, Jorney LJ. Neuraminidase-induced thrombocytopenia in rats. Br J Haematol. 1972;22:93-101.

41. Grottum KA, Jeremic M. Neuraminidase injections in rabbits. Reduced platelet surface charge, aggregation and thrombocytopenia. Thromb Diath Haemorrh. 1973;29:461-9.

42. Sakr $Y$, Vincent $J \mathrm{~L}$, Ruokonen $\mathrm{E}$, et al. Sepsis and organ system failure are major determinants of post-intensive care unit mortality. J Crit Care. 2008:23:475-83

43. Ospina-Tascon GA, Buchele GL, Vincent JL. Multicenter, randomized, controlled trials evaluating mortality in intensive care: doomed to fail? Crit Care Med. 2008;36:1311-22.

44. Cooper DJ, Rosenfeld JV, Murray L, et al. Decompressive craniectomy in diffuse traumatic brain injury. N Engl J Med. 2011;364:1493-502.

45. von Hundelshausen P, Weber C. Platelets as immune cells: bridging inflammation and cardiovascular disease. Circ Res. 2007:100:27-40.

\section{Submit your next manuscript to BioMed Central and we will help you at every step:}

- We accept pre-submission inquiries

- Our selector tool helps you to find the most relevant journal

- We provide round the clock customer support

- Convenient online submission

- Thorough peer review

- Inclusion in PubMed and all major indexing services

- Maximum visibility for your research

Submit your manuscript at www.biomedcentral.com/submit
) Biomed Central 